\title{
Internal Medicine Clerkship Director: a Title Whose Role Is Changing
}

\author{
Shobhina G. Chheda, MD MPH' and Kimberly Tartaglia, $M D^{2}$ \\ 'Department of Medicine, University of Wisconsin School of Medicine and Public Health, Madison, WI, USA; ${ }^{2}$ Department of Medicine, Ohio State \\ University College of Medicine, Columbus, OH, USA.
}

$\mathrm{J}$ Gen Intern Med 35(5): 1366-7

DOI: $10.1007 / \mathrm{s} 11606-020-05756-8$

(c) Society of General Internal Medicine 2020

$\mathrm{T}$ he clerkship director role is often considered the face of undergraduate medical education for departments of internal medicine. This likely originates from the time where the greatest point of contact between medical students and the department was the classic third-year medical student internal medicine clerkship overseen by the quintessential clerkship director. However, undergraduate medical education is evolving and is challenged to incorporate new content within new structures, implement new teaching and assessment methods based on best practices, address changing expectations of learners, and engage in continuous quality improvement to meet Liaison Committee on Medical Education (LCME) accreditation standards ${ }^{1-4}$. Many are curious about how this change is impacting clerkship directors, our leaders of medical student education within departments of medicine, and about what we need to do to ensure ongoing future success of internal medicine medical student education.

In this issue of JGIM, Glod et al. surveyed internal medicine clerkship directors at LCME-accredited medical schools with membership in Clerkship Directors of Internal Medicine (CDIM) ${ }^{5}$. The 2017 survey was designed to outline roles and responsibilities of clerkship directors as well as describe the support for clerkship directors, both in time and financial resources. With a robust response rate $(83 \%, 107 / 129)$, this study provides a glimpse into the life of the internal medicine clerkship director and may provide insights into medical student education more broadly. Not surprisingly, clerkship directors still oversee the core clerkship inpatient experience (94\%), but $70 \%$ were responsible for more than one experience with a mean of 2.4 clinical experiences, including outpatient, electives, and sub-internships ${ }^{5}$. Thus, although the clerkship director has remained the pivotal point of contact between medical students and the department of medicine, they now often directly oversee multiple curricular experiences. Additionally, beyond the traditional eleven responsibilities of a clerkship director defined almost two decades ago, there are now additional areas that clerkship directors have

Published online March 10, 2020 identified as key responsibilities ${ }^{6}$. These include the following: the recruitment of new faculty and sites, addressing student mistreatment reports, attending departmental and medical school curriculum meetings, ensuring compliance with LCME accreditation standards, faculty development, and training and managing additional faculty leaders ${ }^{5}$. These findings were similar to those obtained when surveying clerkship directors from a variety of disciplines who were in attendance at a national AAMC meeting in $2016^{7}$.

This study also provides data on the team-based nature of clinical education. Eighty-three percent $(n=86)$ of respondents reported sharing oversight of internal medicine clinical experiences with other faculty, and interestingly $76 \%(n=65)$ reported that the other faculty, with titles such as clerkship codirectors, inpatient clerkship directors, ambulatory clerkship directors, and site directors, reported directly to the clerkship director ${ }^{5}$. Another piece of complexity highlighted by this study is the variability to whom clerkship directors report. In their role as clerkship directors, the majority reported within the department to either the department chair $(31 \%, n=54)$ or another department leader (e.g., vice chair) $(24.7 \%, n=43)$; however, a significant number $(40.2 \%, n=70)$ reported to the dean of curriculum (or equivalent) at the medical school ${ }^{5}$. This study suggested that for many this was in contrast to whom they reported for their greatest funded, or primary academic role, which often was not their clerkship director role. Thus, clerkship directors are now more likely to be midlevel leaders and managers of teams of medical student educators and have matrix reporting structures within and external to the department.

As one would expect, with these new responsibilities and complexities should come additional resources. The study authors found that less than one-third of clerkship directors $(n=33)$ reported having $41 \%$ or more full-time equivalent (FTE) support and 15\% ( $n=16)$ reported having less than $20 \%$ FTE support ${ }^{5}$. This is in contrast with the 50\% FTE support recommended in the 2003 guidelines regarding expectations for clerkship directors ${ }^{6}$. Notably these guidelines did not account for the additional resources recommended for those running internal medicine sub-internships, a co-role we see $42 \%$ of these respondents directly accountable for ${ }^{5,8}$. Of the $83 \%(n=87)$ of clerkship directors that report additional faculty to assist with internal medicine clinical experiences, the median of support is 1.0 FTE; however, there is significant 
variation by role and institution ${ }^{5}$. Without specifically mapping FTE to varying roles and responsibilities for medical student education within the department and assigning an adequate FTE for team leadership, it is challenging to determine the extent of adequacy of total FTE within departments of medicine for undergraduate medical education. Beyond time, only $35 \%(n=38)$ of clerkship directors had a budget for managing their clerkship, yet this has been a recommendation in past guidelines ${ }^{5,6}$. It is, however, unclear whether or not lack of a budget equates with lack of financial resources for clerkship directors, or if these resources are covered through financial resources provided centrally by departments or schools of medicine.

A concerning result that authors shared is from a glimpse into the 2018 CDIM survey data, where $25 \%$ of respondents reported symptoms of burnout, and 35\% related that they were likely to resign in the next year ${ }^{5}$. Though possible reasons for burnout were not discussed, it does raise attention to a potential discrepancy between responsibilities of CDs and the support and resources offered to them. Previous studies have shown that there is often a mismatch between what department chairs perceive as barriers and needs compared with leaders of medical student experiences in internal medicine ${ }^{9}$. It is vital that there is partnership between department chairs, vice chairs, and clerkship directors so that local needs can be more fully understood, and department chairs/vice chairs can collaborate with deans of schools of medicine to ensure adequate resources are available.

Without question, further research is needed to more specifically define the time and resources needed to effectively fulfill various responsibilities within undergraduate medical education. One size will not fit all, and care should be taken to ascertain which responsibilities translate to fixed effort and for which effort is variable by number of students, number of sites, number and/or duration of rotations, or other factors. The defined FTE required by the Accreditation Council for Graduate Medical Education (ACGME) for resident training should be considered while creating benchmarks for medical student education training. Additionally, identifying the skill sets needed to successfully accomplish tasks, including leadership responsibilities, and develop expertise in the educational mission should further inform budgetary needs for professional development.

As we wait for additional research outcomes, we must also think about the future. Just as the ACGME has announced its strategic planning process entitled Medicine 2035, undergraduate medical educators must also anticipate the future needs of the patients, the health system, and physicians-in-training ${ }^{10}$. Internists are recognized for insights and clinical judgment and the responsibility of medical schools to ensure this competency in their graduates will increase as will the call for internists to participate in this education ${ }^{10}$. Departments of medicine have always played a crucial role in educating medical students and, with these new needs, are well positioned to lead in creating the physicians of 2035. To do so, focus on medical student education is prerequisite and will require an investment in the key education faculty who design, implement, and assess this future curriculum and mentor our future physicians - an investment in the "clerkship director" and their teams.

Corresponding Author: Shobhina G. Chheda, MD MPH; Department of Medicine University of Wisconsin School of Medicine and Public Health, Madison, WI, USA (e-mail: sgc@medicine.wisc.edu).

\section{Compliance with Ethical Standards:}

Conflict of Interest: The authors declare that they do not have a conflict of interest.

\section{REFERENCES}

1. Policy Priorities to Improve Our Nation's Health. How Medical Education is Changing. https://www.aamc.org/system/files/c/2/472906howmedicaleducationischanging.pdf Accessed 1 Feb 2020.

2. Ramnanan CJ, Pound LD. Advances in medical education and practice: student perceptions of the flipped classroom. Adv Med Educ Pract. 2017;8:63-73.

3. Fazio SB. Ledford CH. Aronowitz PB et al. Competency-based medical education in the internal medicine clerkship: a report from the Alliance for Academic Medicine undergraduate medical education task force. Acad Med 2018:93(3) 421-427.

4. Liaison Committee on Medical Education. Functions and Structures of a Medical School 2020. https://lcme.org/publication/\#standards. Accessed 1 Feb 2020.

5. Glod SA, Alexandraki I, Jasti H, et al. Clerkship roles and responsibilities in a rapidly changing landscape: a national survey of internal medicine clerkship directors. J Gen Intern Med. 2020; https://doi.org/ 10.1007/s11606-019-05610-6.

6. Pangaro L, Bachicha J, Brodkey A, et al. Expectations of and for clerkship directors: a collaborative statement from the Alliance for Clinical Education. Teach Learn Med 2003;15(3):217-222.

7. Beck Dallaghan GL, Ledford CH, Ander D, et al. Evolving roles of clerkship directors: have expectations changed? Medical Education Online. 2020;25:1714201

8. Aiyer M, Appel $\mathbf{J}$, Fischer $\mathbf{M}$, et al. The role of the internal medicine subinternship director in the 21st century. Am J Med 2008;121(8) 733737.

9. Fazio SB, Shaheen AW, Amin AN. Tackling the problem of ambulatory faculty recruitment in undergraduate medical education: An AAIM position paper. Am J Med 2019;132(10):1242-1246.

10. Nasca TJ, Thomas CW. Medicine in 2035: Selected insights from ACGME's scenario planning. J Grad Med Educ. 2015;7(1):139-142.

Publisher's Note Springer Nature remains neutral with regard to jurisdictional claims in published maps and institutional affiliations. 